\title{
Perspectiva y construcción teórica en enfermería
}

\author{
Araceli Jiménez Mendoza,* Hugo Tapia Martínez **
}

\begin{abstract}
RESUMEN
El ejercicio de la teoría desarrolla conocimientos que ayudan a la expansión y el desarrollo de la ciencia. Esto despierta en los hombres el interés por el conocimiento científico para dar respuesta a incógnitas y problemas diversos. La elaboración y aplicación de nuevas teorías ha generado avances científicos en beneficio de la humanidad en aquellas comunidades y países que han invertido para ello; por el contrario, los países pobres se han visto limitados en estos beneficios. Aunque se utilizan a partir de la razón teórica, se pueden generar nuevas formas de pensar o de relacionarse con la realidad para explicar, problematizar o formar juicios en relación al cuidado como objeto de estudio de enfermería.
\end{abstract}

Palabras clave: Construcción, enfermería, teoría.

\section{Theoretical perspective and nursing building}

\begin{abstract}
Based on the theories, have developed expertise that have enabled the growth and development of science. This allows men to develop interest in the scientific knowledge to answer many unknowns and challenges. The circumstances have allowed the construction and application of new theories and scientific advances have led to the benefit of humanity in communities and countries that have invested in it; however, poor countries have been limited to these privileges to be expensive the different dimensions of humanity, although used from theoretical reason can generate new ways of thinking, or of relating to reality to explain, problematize or make judgments regarding care as an object of study in nursing.
\end{abstract}

Key words: Construction, nursing, theory.

\section{INTRODUCCIÓN}

$\mathrm{A}$ partir de la teoría, los hombres han desarrollado el conocimiento que permite la expansión y el desarrollo de la ciencia. Bajo este conocimiento se interpreta lo desconocido en términos de lo ya conocido y es a partir de las teorías que los hombres han desarrollado el conocimiento científico para dar respuesta a incógnitas y problemas diversos.

En la realidad, la construcción de nuevas teorías ha generado avances científicos que si bien han beneficiado a la humanidad, sobre todo en países que han invertido para

\footnotetext{
* Licenciada en Enfermería y Obstetricia, Maestría en Ciencias de la Enfermería, Profesora de Carrera Titular A, Coordinadora de Intercambio Académico y Movilidad Estudiantil.

** Licenciado en Enfermería y Obstetricia, Maestro en Ciencias de Enfermería, Profesor de Carrera Asociado B, Área Proceso Salud Enfermedad en la Reproducción Humana.

ENEO-UNAM. Doctorado Itinerante en Ciencias de Enfermería. Universidad Nacional de Trujillo Perú/Escuela Nacional de Enfermería y Obstetricia UNAM.

Correspondencia: Araceli Jiménez Mendoza. Camino Viejo a Xochimilco y Viaducto Tlalpan sin número, Col. San Lorenzo Huipulco, Delegación Tlalpan, 14370, México,D.F.E-mail: ajimenez55070@yahoo.com 
ello en beneficio de sus comunidades, por el contrario, los habitantes de países pobres se han visto limitados de esos avances.

En ese tenor, enfermería, como disciplina en proceso de consolidación, ha ido construyendo su desarrollo científico disciplinario, partiendo del conocimiento empírico por excelencia; en la búsqueda por superar la dependencia de sustentos teóricos procedentes de otras disciplinas se ha enfocado en la definición de su objeto de estudio, de sus constructos teóricos disciplinarios y de la conformación de sus comunidades científicas.

Para enfermería, es imprescindible contar con teorías que expliquen su objeto de estudio, con el o los paradigmas que en ese momento histórico imperen en la comunidad científica. Son diversas las teorías que se han desarrollado en enfermería; algunas suelen explicar de manera general el objeto de estudio (macroteorías) y otras se dedican a estudiar algunas partes del cuidado (teorías intermedias).

Al paso del tiempo, y al mirar en retrospectiva, se han asumido algunas teorías más a manera de moda que por su valor científico; es decir, en cuanto se difunde alguna teoría, independientemente del contexto en el que se originó, se busca aplicarla en cualquier área del cuidado, sin haber considerado los criterios de validez que debe cumplir.

En este ensayo se analizarán, en perspectiva y prospectiva, las teorías científicas de enfermería y el concepto científico como sustantivo para su construcción. Si bien las diferentes aportaciones teóricas han sido valiosas y aluden a un sustento filosófico, epistemológico y técnico, no siempre han dado respuesta a los cuestionamientos: ¿En qué medida las teorías de enfermería abarcan el estudio sobre el cuidado? ¿Tienen algún enfoque epistemológico? Si no es así, ¿qué debemos hacer para alcanzarlo?

\section{DISCUSIÓN}

Los autores mencionan que todas las teorías cumplen el requisito de contar con conceptos; para Kerlinger, una teoría es un conjunto de constructos (conceptos), definiciones y proposiciones, relacionados entre sí, que presentan una visión sistemática de fenómenos especificando relaciones entre variables con el propósito de explicar y predecir los fenómenos (Hernández et al. 2003). Pollit y Hungler refieren que las teorías científicas comprenden un conjunto de proposiciones vinculadas con la interrelación entre conceptos, de los cuales se puede deducir una gran cantidad de observaciones empíricas (Pollit-Hungler, 1997). Por lo tanto, las teorías científicas describen, explican y predicen con el fin de ampliar el conocimiento de una ciencia.

En el proceso de construcción del conocimiento, la teoría constituye el instrumento a partir del cual se logra el ra- zonamiento y nuevas formas de pensar. En este sentido, la teoría constituye lo que Zemelman ha denominado "la razón teórica", que implica la transmisión de conceptos y propicia la vinculación con la sociedad (Zemelman, 1975). La razón teórica exige el uso de conceptos para arribar a la enunciación del conocimiento organizado. A partir de la razón teórica se pueden generar nuevas formas de pensar o de relacionarse con la realidad para explicar, problematizar o formar juicios.

En este discurrir del pensamiento influyen aspectos culturales, ideológicos y dogmáticos (Eusse, 1994). De acuerdo con estos autores, la construcción de las teorías o formas de pensar no sólo requiere de organizar, relacionar y sistematizar los conceptos que las forman, para explicar la realidad, sino de ubicar el entorno socio-histórico en el cual se desarrollan, de manera que estén en concordancia o formen parte del paradigma epistémico predominante.

Las teorías a menudo se transforman en paradigmas debido a que la comunidad científica asume ciertos contenidos de manera subjetiva, sin analizar el problema; de tal forma que al adoptar la teoría se parte del referente, producto de su proceso de racionalidad. Khun señala que cuando las explicaciones de la teoría no satisfacen los fenómenos de la naturaleza (se encuentran anomalías), el paradigma predominante en la ciencia normal entra en contradicción con nuevas teorías rivales que lo intentan explicar. Si alguna de estas teorías rivales logra explicarlo y además logra explicar otros fenómenos, impera, cambiando de paradigma y se logra un nuevo capítulo de ciencia normal; este proceso ocurre en todas las ciencias, a través de la historia (Khun, 1986). El paradigma que aún prevalece es el empirismo lógico, que en términos de una teoría aceptada como verdad debe tener consistencia entre sus componentes, conceptos y método científico. Aunque también existen otras doctrinas filosóficas que ponen en entre dicho este paradigama.

Para Popper, las teorías son conjeturas, hipótesis generales que permiten explicar los fenómenos. Nunca son verdaderas, pero sí pueden ser falseadas, lo cual debe encaminar al científico a rechazarlas. Las teorías son redes que lanzamos para apresar aquello que llamamos el "mundo": para racionalizarlo, explicarlo y dominarlo (Popper, 1959). El falsacionista considera que la ciencia es un conjunto de hipótesis que se proponen, con el propósito de describir o explicar de manera precisa el comportamiento de algún aspecto de la naturaleza. Sin embargo, no todas las hipótesis lo consiguen.

Una condición fundamental que debe cumplir cualquier hipótesis para conseguir el estatus de teoría o ley científica es que sea falseable o refutable; es decir, las teorías científicas no se verifican: se refutan. Aunque según sus críticos, la refutación misma podría ser falsa, ya que los científicos no creen en la falsedad de sus teorías. 
Por su parte, Feyeraben y en concordancia con Popper en contra del principio de empiricismo, indica: "Es posible iniciar el trabajo científico formulando hipótesis que contradigan teorías sólidamente confirmadas corroboradas hasta ese momento; podemos ganar una nueva perspectiva que la teoría dominante no permitía debido al requisito de consistencia entre hipótesis y teoría."

Consecuentemente, crece el número de teorías que Feyeraben llamó pluralismo teórico, el cual señala que es benéfico para la ciencia, mientras que la uniformidad teórica de los positivistas favorece el dogmatismo e inutiliza la crítica de los científicos. Entre más teorías se generen y se contradigan, mayor conocimiento se obtiene de la realidad.

Una vez considerado un panorama de las aportaciones de Zemelman, Khun, Popper y Feyerabend hacia las teorías, es necesario mencionar otros aspectos a considerar en la construcción de teorías: aquellos relacionados con el sujeto como constructor, es decir, los requisitos o pasos del investigador que intenta elaborar una teoría.

La construcción de teorías involucra dos procesos: el psicológico-cognitivo y el lógico-metodológico (Pérez M. 2010), es decir, el intelecto y en particular nuestro talento se hace posible gracias a la construcción de teorías.

El primer paso inicia con un plan de acción personal que incluye la búsqueda de todo aquel material de lectura necesario para discernir, interpretar, discutir y dilucidar los conocimientos que se tienen y los que haya que complementar en referencia a lo que es una teoría. No deben quedar brechas de conocimiento, pues más adelante serán obstáculos epistemológicos. Tal como se ha sugerido en nuestro Doctorado Itinerante. Y continúa la autora:

El segundo paso es la organización del material de lectura, las ideas que vayan surgiendo en torno al proceso de construcción de teorías, los pensamientos creativos y el tiempo. El siguiente paso es la dedicación, si ya hemos preparado lo necesario y hemos organizado inclusive el tiempo, es el momento de seguir los horarios seleccionados y dedicarse a leer, escribir, pensar, discutir, interactuar con su material de estudio y lectura, con sus amigos que están construyendo teorías y consigo mismo.

El paso que sigue es la evaluación, que puede darse, cuando se estime necesario, en forma de registro de los avances alcanzados, los logros obtenidos y en función del desarrollo de su plan. El paso final es el replanteamiento; en este paso se reflexiona sobre el estilo personal de desarrollar todo el plan, sobre la administración del tiempo, los logros alcanzados y la satisfacción personal con todo lo planteado y alcanzado. Más vale hacer menos, pero hacerlo bien, que hacer más pero mal, es decir, sin producir nada. La única persona que conoce sus limitaciones y la única capaz de superarlas es uno mismo.
Como podemos apreciar hasta aquí, las teorías y su construcción se enmarcan en un proceso histórico para su pertinencia, sirven para explicar y predecir los fenómenos de la realidad, tienen como fin generar conocimientos que consoliden a las ciencias como tales, pueden obedecer a una o varias posturas epistemológicas y para quien trate de construir una teoría se requiere de dos procesos: el psicológicocognitivo y el lógico-metodológico. Ahora analizaremos lo que sucede en enfermería. En efecto, enfermería cuenta con un vasto bagaje teórico, principalmente norteamericano, que ha permitido su desarrollo.

En la disciplina de enfermería han surgido una serie de teorías, principalmente después de los años 50 del siglo $\mathrm{XX}$, las cuales se han asumido como únicas opciones y se han constituido en ideologías dominantes por ya largo tiempo, generando resistencia a la búsqueda de nuevos paradigmas teóricos de enfermería no sólo para la comprensión de fenómenos, sino también para transformar su práctica profesional en torno a la salud.

Asumimos que la enfermera debe siempre contar con una(s) teoría(s) que sustente(n) su quehacer profesional, es decir, primero que piense lo que va a hacer y después hacerlo. Sin embargo, en la práctica profesional común, a menudo la enfermera ejecuta sus "cuidados", como valorar signos vitales, somatometría, tendido de cama, aspiración de secreciones, ministración de medicamentos, etc., sin pensar en qué medida vincula dichas intervenciones con una postura teórica y su método, o si la toma como sustento, ésta es de carácter dogmático sin posibilidad de pensamiento crítico que genere nuevo conocimiento.

Se ha dicho que enfermería es una ciencia o disciplina emergente y joven, que sus teorías y/o modelos aplicados a su metaparadigma y su método son singulares, es decir, que tienen limitaciones, y que por esta razón no puede ser falseada; que tiene hipótesis en sus teorías, pero no hipótesis que las refuten, a diferencia del racionalismo crítico que se mueve en la universalidad. También hay mucho conocimiento por descubrir en su quehacer, por ejemplo con el enfoque cualitativo al interpretar el significado de saberse con VIH positivo en mujeres embarazadas.

\section{CONSIDERACIONES FINALES}

John Bernal, en 1981, planteó el fenómeno de la convergencia general de las ciencias, en el que la ciencia misma se vería afectada por la superposición y extensión de los campos, lo que implica la interacción entre los diversos dominios de la ciencia, la tecnología y la política.

La convergencia existe en el espacio y en el tiempo, de ahí el planteamiento de las transdisciplinas, por lo cual existe la posibilidad, de acuerdo a Popper y a Kuhn, de que no 
sean propuestas absolutas, sino provisionales, de tal forma que es el momento de establecer acciones creativas con prospectiva, ya que tales esfuerzos nos conducen a lo que denomina J Bernal (1981) "Planeación de la ciencia"; esto es, hacer "ciencia de la ciencia" de manera sistemática.

En el escenario previo se plantea que en la evolución de la ciencia se han construido teorías con diversos enfoques, las que a su vez han sido replanteadas o sustituidas por otras; en ese transcurso fueron transmitidas y constituidas como normas ideologías que han asegurado la continuidad de ciertos grupos sociales con derechos y privilegios, muchas veces $\sin$ ser verificadas.

Con base en lo anterior, a enfermería le corresponde impulsar la enseñanza y formación de una comunidad científica madura, creativa, comprometida y responsable que provea de teorías claras, precisas, sencillas y sólidas que den sustento a la disciplina, pero también dinámica para transformarse y juiciosa para no subsumir $i$ ? de nuevo en la interdependencia científica estableciendo fronteras.

Los conocimientos disciplinarios son producto de las relaciones entre la teoría, la práctica y la investigación, y entre más prolífera sea esta última, mayor valor se le da a la profesión de enfermería. Ante nuevos conocimientos de la teoría y la investigación, habrá mejores descripciones explícitas e interpretaciones de sus resultados cualitativos o cuantitativos. Nuestra tarea es promover el desarrollo de la ciencia de enfermería. El progreso teórico disciplinario es un componente imprescindible para el profesional de enfermería.

\section{BIBLIOGRAFÍA}

1. Bernal JD. La ciencia en nuestro tiempo. UNAM. Ed. Nueva Imagen. México, 1981.

2. Bernal JD. La ciencia en la historia". UNAM. Ed. Nueva Imagen. México 1981.

3. Eusse ZO. Proceso de construcción del conocimiento y su vinculación con la formación docente. Perfiles educativos, enero-marzo, No. 63. UNAM, México, D.F. 1994. Disponible en: http://redalyc.uaemex.mx/ redalyc/pdf/132/13206305.pdf consultado el 02 de abril de 2012.

4. Feyerabend P. Tratado contra el método. Madrid. Editorial Tecnos. 1986.

5. Hernández SR, Fernández CC, Baptista LP. Metodología de la investigación. Ed. McGraw-Hill Interamericana. México, D.F. 2003.

6. Kuhn TS. La estructura de las revoluciones científicas. México: FCE. 1986.

7. Polit DF, Hungler BP. Investigación científica en ciencias de la salud. Ed. McGraw-Hill Interamericana. México, D.F. 1997.

8. Popper, Karl R. La lógica de la investigación científica. 5a Reimpresión. Ed. Tecnos. Madrid. 1980.

9. Popper, Karl R. Conjeturas y refutaciones. Ed. Paidós. Barcelona. 1994, 1972.

10. Zemelman H. Uso crítico de la teoría en: En torno a las funciones analíticas de la totalidad 1. Universidad de la Naciones Unidas, El Colegio de México. México. 1975. 